\title{
Assessment of the diagnostic value of CEA, CA125, and CRP and their cut-off point for discrimination of exudative pleural effusions
}

Hanie Raji1', Seyed Hamid Borsi ${ }^{1}$, Mehrdad Dargahi MalAmir ${ }^{1}$, Ahmad Reza Asadollah Salmanpour* DOI. 10.21931/RB/2021.06.03.10 Abstract: Pleural effusion is divided into exudative and transudative effusion, and the distinction between exudate and transudate requires multiple investigations of biochemical parameters and their comparison in pleural fluid and serum. This study aimed to assess the diagnostic value of CEA, CA125, and CRP and their cut-off point for discrimination of exudative pleural effusions. This epidemiological and cross-sectional study was performed on 50 patients aged between 18 to 90 years with the diagnosis of exudative pleural effusion referred to Imam Khomeini Hospital in Ahvaz in 2018 and 2019. Demographic and clinical information of patients were collected. The pleural effusion was diagnosed based on physical examination and chest radiography. Pleural effusion was confirmed by thoracentesis. A pleural fluid sample was taken from all patients, and the levels of CEA, CA125, and CRP markers were measured in the pleural fluid. Differentiation of transudate and exudate pleural effusions was performed using Light criteria. The mean CEA and CA125 level of pleural fluid were significantly higher, and the mean CRP level of pleural fluid was significantly lower in patients with malignant diagnoses $(P<0.05)$. Cut-off value with highest sensitivity and specificity in differentiating types of exudative pleural effusions was obtained for CEA tumor marker (greater than 49.8), CA125 tumor marker (greater than 814.02), and CRP marker (less than 7.56). Also, in differentiating types of exudative pleural effusions, CEA tumor marker had sensitivity (89.03\%) and specificity (78.42\%); CA125 tumor marker had sensitivity (53.18\%) and specificity (62.44\%), and CRP marker had sensitivity (82.16\%), and specificity (89.05\%) were. Although the tumor markers had high specificity in the present study, the low sensitivity of some of these tumor markers reduced their diagnostic value. On the other hand, given the numerous advantages of tumor markers, such as low cost and non-invasive, combining them with another can increase the diagnostic value and accuracy.

Key words: Pleural effusion, Exudative, Transudative, Tumor markers.

\section{Introduction}

Pleural effusion is one of the most common clinical manifestations associated with some chest diseases ${ }^{1}$, which is the accumulation of fluid in the pleural cavity and is often caused by a systemic or intrathoracic process. The prevalence varies by clinical setting, but $90 \%$ of all pleural effusions are caused by heart failure, malignant processes, and pneumonia and can lead to serious health problems if not properly treated or diagnosed $^{2}$. The fluid that enters the pleural space can be of the origin of the pleural capillaries, interstitial lung space, intra-aortic lymphatics, intrathoracic blood vessels, or peritoneal cavity ${ }^{3,4}$. The pleural effusion is divided into two types of exudative and transudative effusions ${ }^{3,5}$. The distinction between exudate and transudate requires multiple investigations of biochemical parameters and their comparison in pleural fluid and serum ${ }^{6-9}$. Recently, to help differentiate the etiologies of pleural effusion, several studies have investigated tumor markers as a potential alternative to invasive procedures ${ }^{10-13}$. Different studies have investigated the diagnostic value of different tumor markers to differentiate different types of pleural effusion. However, the wide range of sensitivity, specificity, and cut-off values and the inconsistency in the results have made their diagnostic accuracy still questionable ${ }^{14-18}$. CEA has been the most common marker tumor studied for the diagnosis of malignant pleural effusion ${ }^{19}$. It has recently been reported that serum and fluid levels of pleural effusion CA-125 can be used to diagnose pleural effusion malignancy ${ }^{20,21}$. In addition, CRP is often produced by the liver, and CRP levels in pleural effusions can be used to differentiate parapneumonic effusions from other types of effusions ${ }^{22,23}$. Since no high sensitivity and specificity, the marker has been identified for the diagnosis of exudative effusion pleural effusions and due to inconsistency in the results of our studies, the aim of the present study was to evaluate the biomarker value of CEA, CA-125 and CRP tumor biomarkers in differentiation between exudative effusion pleural effusions.

\section{Materials and methods}

\section{Study designs}

Following approval of the study in the Ethics Committee of Ahvaz Jundishapur University of Medical Sciences (Code of Ethics: IR.AJUMS.REC.1397.950), this study is an epidemiological and cross-sectional study on 50 patients aged 18 to 90 years with the diagnosis of exudative pleural effusion who referred to Imam Khomeini Hospital in Ahvaz in 2018 and 2019. Initially, the goals, benefits of participating in the study, and how to conduct the research were explained to participants. Eligible patients were then enrolled in the study, if desired, with written consent.

At first, demographic data and clinical history of all patients were obtained and collected in a checklist. Required information about the underlying disease and the cause of pleural effusion was also collected and recorded based on the patient's medical record findings. Patients with exudative pleural effusion with different etiologies were included in the study, and biomarkers were measured before any treatment. Patients with unknown pleural effusion origin were excluded.

The diagnosis of pleural effusion was made after a physical examination and chest imaging. Pleural effusion was confirmed by thoracentesis. Microbiological, biochemical, and cytological studies were also performed for all patients. Di-

\footnotetext{
${ }^{1}$ Air Pollution and Respiratory Diseases Research Center, Faculty of Medicine, Ahvaz Jundishapur University of Medical Sciences, Ahvaz, Iran.
} 
fferentiation of transudate and exudate pleural effusions was performed using light criteria. Accordingly, the presence of one of the following indicated exudative pleural effusion.

- Pleural protein/serum protein ratio $>0.5$

- Serum LDH/pleural LDH ratio > 0.6

- Pleural LDH activity exceeds two-thirds of the highest normal level for serum $\operatorname{LDH}^{10,24,25}$.

A pleural fluid sample was obtained from all patients, and the supernatants were collected after centrifugation (3500 rpm for 10 minutes) and stored at $-20^{\circ} \mathrm{C}$ for final testing. The pleural fluid sample was inserted through a needle between the rib cage based on examination and percussion, and auscultation (sound reduction and dullness). The samples were sent to the laboratory after collection and the levels of CEA, CA125, and CRP markers in the pleural fluid of the patients were measured.

CEA and CA125 measurements were performed on pleural specimens by electrochemical luminescence (ECL) and CRP measurements by turbidometric technique. All biomarkers were analyzed according to the manufacturer's instructions. Finally, all data collected were analyzed statistically to evaluate the diagnostic value of each tumor marker in different types of exudative pleural effusion.

\section{Statistical analysis}

The data are analyzed by descriptive statistics, including mean, standard deviation, frequency, and percentage. Data were analyzed using the Kolmogorov-Smirnov test and Q-Q plot and variance homogeneity by Leven test. Independent t-test (or Mann-Whitney nonparametric test), chi-square (or Fisher exact test), and logistic regression were used for data analysis. The ROC curve was used to determine the diagnostic value of tumor markers, and the area under the ROC diagram (AUC) was considered as the diagnostic value of the biomarker. Sensitivity, specificity, accuracy, positive and negative predictive values of each tumor marker were also calculated. All analyses were performed using SPSS software version 22, and the significance level was considered less than 0.05 .

\section{Results}

The distribution of patients by gender was approximately similar, and the percentage of male and female patients was similar (48\% vs. 52\%). Patients older than 60 years had the highest frequency (72\%) compared to other age groups. The etiology of the disease in most patients was adenocarcinoma and parapneumonic (Table 1).

To determine the diagnostic value of CEA, CA125, and CRP markers in differentiating different types of exudative pleural effusions, patients were divided into three groups according to the etiology of the disease. The mean level of CEA in pleural fluid was significantly higher in patients with malignancy ( $P$ $<0.05)$. This rate was also higher in patients with the etiology of tuberculosis than in patients with the parapneumonic diagnosis. The mean CA-125 level of pleural fluid was significantly higher in patients with malignancy $(P<0.05)$. This rate was also higher in patients with the etiology of tuberculosis than in patients with the parapneumonic diagnosis. The mean pleural fluid CRP level was significantly lower in patients with malignancy $(P<0.05)$. Also, this rate was lower in patients with tuberculosis than in patients with parapneumonic diagnosis (Table 2).

The cut-off value with the highest sensitivity and specificity for CEA tumor marker differentiation in exudative pleural effusions was more significant than 49.8. According to this cut-off value, the sensitivity of the CEA tumor marker in differentiating different types of exudative pleural effusions was $89.03 \%$, specificity was $78.42 \%$, positive predictive value was 82.01 , and negative predictive value was $64.36 \%$.

The cut-off value with the highest sensitivity and specificity for CA125 tumor marker in the differentiation of exudative pleural effusions was greater than 814.02. Accordingly, CA125 tumor marker sensitivity in differentiating pleural effusions was $53.18 \%$, specificity was $62.44 \%$, positive predictive value was $67.34 \%$, and negative predictive value was $59.19 \%$.

The value of highly sensitive and specific cut-off for the

\begin{tabular}{|c|c|c|}
\hline \multicolumn{2}{|c|}{ Variables } & N (\%) \\
\hline \multirow{3}{*}{ Gender } & Female & $24(48 \%)$ \\
\cline { 2 - 3 } & Male & $26(52 \%)$ \\
\hline \multirow{4}{*}{ Age (year) } & $\mathbf{1 5}-\mathbf{2 9}$ & $1(2 \%)$ \\
\cline { 2 - 3 } & $\mathbf{3 0 - 4 4}$ & $5(10 \%)$ \\
\cline { 2 - 3 } & $\mathbf{4 5 - 5 9}$ & $8(16 \%)$ \\
\cline { 2 - 3 } & $\mathbf{6 0 - 7 4}$ & $22(44 \%)$ \\
\cline { 2 - 3 } & $\mathbf{7 5 - 9 0}$ & $14(28 \%)$ \\
\hline \multirow{5}{*}{ Etiology } & Para pneumonic & $18(36 \%)$ \\
\cline { 2 - 3 } & Adenocarcinoma & $27(54 \%)$ \\
\cline { 2 - 3 } & Mesothelioma & $2(4 \%)$ \\
\cline { 2 - 3 } & Tuberculosis & $3(6 \%)$ \\
\hline
\end{tabular}

Table 1. Demographic information of the patients. 


\begin{tabular}{|c|c|c|c|}
\hline Group & $\begin{array}{c}\text { Pleural fluid CEA } \\
\text { (mg/l) }\end{array}$ & $\begin{array}{c}\text { Pleural fluid CA125 } \\
\text { (mU/mL) }\end{array}$ & $\begin{array}{l}\text { Pleural fluid } \\
\text { CRP (mg/l) }\end{array}$ \\
\hline Malignancy & $46.11 \pm 35.80$ & $782.45 \pm 314.05$ & $6.12 \pm 2.89$ \\
\hline Parapneumonic & $4.67 \pm 2.09$ & $351.32 \pm 189.14$ & $21.56 \pm 11.83$ \\
\hline Tuberculosis & $11.32 \pm 5.45$ & $544.19 \pm 264.10$ & $17.02 \pm 5.31$ \\
\hline P-Value & $0.008^{*}$ & $0.007^{*}$ & $0.001^{*}$ \\
\hline
\end{tabular}

Table 2. Comparison of pleural fluid CEA levels based on disease etiology.

CRP marker differentiating different types of exudative pleural effusions was less than 7.56. Accordingly, CRP marker sensitivity in differentiating pleural effusions was $82.16 \%$, specificity was $89.05 \%$, positive predictive value was $64.32 \%$, and negative predictive value was $59.71 \%$.

\section{Discussion}

Malignant pleural effusion is a common problem in cancer patients that can be both a symptom and a complication of a previously diagnosed malignancy. Despite the combination of pleural fluid cytology and pleural biopsy, it is not possible to obtain a diagnosis in many cases ${ }^{24}$. Researchers have been researching to evaluate the value of pleural fluid analysis in the differential diagnosis of pleural effusions on different tumor markers ${ }^{24,25}$.

In our study, 26 patients were male, and 24 were female. Also, in the present study, out of 50 people studied, 18 patients were parapneumonic, 27 patients were adenocarcinoma, 2 patients were mesothelioma, and 3 patients were tuberculosis.

Based on our results, mean levels of pleural fluid CEA and CA125 were significantly higher in patients with malignancy $(P<0.05)$. It also indicated that the rate is higher in patients with tuberculosis etiology than in patients with the parapneumonic diagnosis. The threshold for identifying exudative pleural effusion with the highest sensitivity and specificity for CEA tumor markers is more remarkable than 49.8. consequently, the sensitivity of the CEA tumor marker was $89.83 \%$, the specificity was $78.42 \%$, the positive predictive value was $82.01 \%$, and the negative predictive value $64.36 \%$, which shows and highlights high biomarker effectiveness in the diagnosis of benign and malignant.

Also, the highest sensitivity and specificity for the cut-off value of the CA125 tumor marker when differentiating types of exudative pleural effusion was greater than 814.02. therefore, the sensitivity of the CA125 tumor marker when differentiating exudative pleural effusions was $53.18 \%$, the specificity was $62.44 \%$, the positive predictive value was $67.34 \%$, and the negative predictive value was $59.19 \%$, as obtained from the study.

Owing to the chronic nature of the disease, the approach on individuals affected by unclassical and even unidentified pleural effusion is applied. Additionally, using this method eases the distinguish of malignant cases of tuberculosis with lower costs of the experiment ${ }^{26}$.

In the study done by Nguyen et al., who determined the diagnostic value of tumor antigens for malignant pleural effusions, the sensitivity and specificity were $54.9 \%$ and $96.2 \%$ for CEA tumor markers and $57.5 \% 92.8 \%$ for CA-125, respectively ${ }^{15}$. In another study done by Zhai et al., who investigated the diagnostic accuracy of CEA and CA-125 tumor markers in the differentiation of malignant pleural effusions, The results indicated that the serum levels of both tumor markers were significantly higher in the types of malignant pleural effusions than in benign pleural effusions. The CEA and CA15-3 levels were more stable than the CA- 125 and CA19-9 tumors. CEA was also the best marker to distinguish between benign and malignant pleural effusions. The sensitivity and specificity of CEA were $84.7 \%$ and $90.9 \%$ in the pleural and $64.0 \%$ and $88.0 \%$ in the serum. The sensitivity and specificity for the CA-125 tumor marker were $49 \%$ and $73.1 \%$ in the pleural and $60.4 \%$ and $54.8 \%$ in the serum ${ }^{14}$. Tozzoli et al. compared the diagnostic value of pleural fluid CEA in patients with pleural effusion with histological findings.

The results indicated that the sensitivity and accuracy of pleural fluid CEA were significantly higher than that of pleural cytology, and the sensitivity of diagnosis of benign and malignant cases was high. They deduced that measuring pleural fluid CEA in patients with unexplained etiology of a pleural effusion is a safe and reasonably priced way for doctors to select patients for further examination. Increased pleural CEA values in patients with pleural effusion with negative cytology indicate the need for further invasive examinations, whereas people with low pleural CEA values should only be examined again $^{12}$. In a study by Antonangelo et al., A comparison of tumor markers in benign and malignant pleural effusions with positive, suspicious, and negative cytology showed that the CEA and CA125 markers were significantly higher in malignant effusions with positive cytology. Only the CA125 marker tumor score was significantly higher in the negative or suspected cytological results than in the benign effusions in the pleural fluid. As a result of this study, it could be shown that a tumor sensitivity and specificity of up to $60 \%$ can be used as a parameter for the assessment of patients with a suspected malignancy or cancer in the history ${ }^{27}$. The results of a study by Shalaby et al. showed that the CA-125 tumor marker was significantly higher in patients with exudative effusion than in patients with transudative effusion. This tumor marker was also more common in malignant effusions than benign effusions and tuberculosis compared to other infections. As a result, the highest CA125 level of pleural fluid was observed in malignancy and then in tuberculosis, and thus the level of this 
marker tumor in the pleural fluid could be used as a diagnostic marker for pleural effusion ${ }^{21}$. In the present study, the mean CRP of pleural fluid was significantly lower in patients with malignancy $(P<0.05)$. This rate was also lower in patients with tuberculosis etiology than in patients with the parapneumonic diagnosis.

The limit with the highest sensitivity and specificity for CRP markers when distinguishing different types of exudative pleural effusions was less than 7.56. Accordingly, the sensitivity of the CRP markers when differentiating pleural effusions was $82.16 \%$, the specificity $89.05 \%$, the positive predictive value $64.32 \%$, and the negative predictive value $59.71 \%$. In a study by $\mathrm{Ji}$ et al. To investigate the role of three markers of procalcitonin (PCT), CRP, and CEA in the differential diagnosis of malignant and benign pleural effusions, the CRP and PCT levels were significantly higher in benign pleural effusions than in malignant cases, while the CEA levels were lower were in benign cases. They concluded that the use of a biomarker is not only suitable for the diagnosis of pleural effusion and is not accurate enough. The combination of pleural CRP, pleural CEA and SPCT can effectively support the diagnosis of pleural effusions ${ }^{1,28}$.

\section{Conclusions}

Based on the present study results, the tumor markers examined in this study had high specificity, but the low sensitivity of some of these tumor markers decreased their diagnostic value. However, since tumor markers in the diagnosis of malignant pleural effusion have many advantages, such as low cost and invasive use, the combination of tumor markers can significantly increase the value and diagnostic accuracy.

\section{Acknowledgment}

This research was supported by grants (APRD-9710) from Air Pollution and Respiratory Diseases Research Center, Ahvaz Jundishapur University of Medical Sciences, Ahvaz, Iran. This study is the residency thesis of Dr. Ahmad Reza Asadollah Salmanpour.

\section{Bibliographic references}

1. Ji M, Zhu X, Dong J, Qian S, Meng F, Gu W, et al. combination of procalcitonin, $\mathrm{C}$-reaction protein and carcinoembryonic antigens for discriminating between benign and malignant pleural effusions. Oncology letters. 2018; 16(2): 1727-35.

2. Trapé J, Sant F, Franquesa J, Montesinos J, Arnau A, Sala M, et al. Evaluation of two strategies for the interpretation of tumour markers in pleural effusions. Respiratory research. 2017; 18(1): 103

3. Na MJ. Diagnostic tools of pleural effusion. Tuberculosis and respiratory diseases. 2014; 76(5): 199-210.

4. Light RW. Pleural diseases. Lippincott Williams \& Wilkins(2007).

5. Light RW. Pleural effusions. Medical Clinics. 2011; 95(6): 1055-70.

6. Ferreiro L, Toubes ME, Valdés L. Contribution of pleural fluid analysis to the diagnosis of pleural effusion. Medicina Clínica (English Edition). 2015; 145(4): 171-77.

7. Braunschweig T, Chung J-Y, Choi CH, Cho H, Chen Q-R, Xie R, et al. Assessment of a panel of tumor markers for the differential diagnosis of benign and malignant effusions by well-based reverse phase protein array. Diagnostic pathology. 2015; 10(1): 1-10.

8. Porcel JM, Light RW. Pleural effusions. Disease-a-month: DM. 2013; 59(2): 29.
9. Thomas R, Lee YG. Causes and management of common benign pleural effusions. Thoracic surgery clinics. 2013; 23(1): 25-42.

10.Gu Y, Zhai K, Shi H-Z. Clinical value of tumor markers for determining cause of pleural effusion. Chinese medical journal. 2016; 129(3): 253

11. Volarić D, Flego V, Žauhar G, Bulat-Kardum L. Diagnostic value of tumour markers in pleural effusions. Biochemia medica: Biochemia medica. 2018; 28(1): 73-83.

12. Tozzoli R, Basso SM, D'Aurizio F, Metus P, Lumachi F. Evaluation of predictive value of pleural CEA in patients with pleural effusions and histological findings: A prospective study and literature review. Clinical biochemistry. 2016; 49(16-17): 1227-31.

13. Yoon DW, Cho JH, Choi YS, Kim J, Kim HK, Zo Jl, et al. Predictors of survival in patients who underwent video囚assisted thoracic surgery talc pleurodesis for malignant pleural effusion. Thoracic cancer. 2016; 7(4): 393-98.

14.Zhai K, Wang W, Wang Y, Liu J-Y, Zhou Q, Shi H-Z. Diagnostic accuracy of tumor markers for malignant pleural effusion: a derivation and validation study. Journal of Thoracic Disease. 2017; 9(12): 5220.

15. Nguyen AH, Miller EJ, Wichman CS, Berim IG, Agrawal DK. Diagnostic value of tumor antigens in malignant pleural effusion: a meta-analysis. Translational Research. 2015; 166(5): 432-39.

16. Gu P, Huang G, Chen Y, Zhu C, Yuan J, Sheng S. Diagnostic utility of pleural fluid carcinoembryonic antigen and CYFRA 21囚1 in patients with pleural effusion: a systematic review and meta खanalysis. Journal of clinical laboratory analysis. 2007; 21(6): 398-405.

17. SHI HZ, LIANG QL, Jiang J, QIN XJ, YANG HB. Diagnostic value of carcinoembryonic antigen in malignant pleural effusion: A meta区 analysis. Respirology. 2008; 13(4): 518-27.

18. Liang Q, Shi H, Qin X, Liang X, Jiang J, Yang H. Diagnostic accuracy of tumour markers for malignant pleural effusion: a meta-analysis. Thorax. 2008; 63(1): 35-41.

19. Hammarström S. The carcinoembryonic antigen (CEA) family: structures, suggested functions and expression in normal and malignant tissues. Seminars in cancer biology 1999; 9(2): 67-81.

20.Shokouhi S, Samanabadi M, Gachkar L. Pleural fluid CA-125 in patients with pleural effusion. Tanaffos. 2005; 4(23-27.

21. Shalaby AED, Moussa HA, Nasr A, Samad MA. A study of CA-125 in patients with pleural effusion. Egyptian Journal of Bronchology. 2015; 9(3): 283-83.

22. Yang Y, Xie J, Guo F, Longhini F, Gao Z, Huang Y, et al. Combination of $\mathrm{C}$-reactive protein, procalcitonin and sepsis-related organ failure score for the diagnosis of sepsis in critical patients. Annals of intensive care. 2016; 6(1): 51.

23. Izhakian S, Wasser WG, Fox BD, Vainshelboim B, Kramer MR. The diagnostic value of the pleural fluid $\mathrm{C}$-reactive protein in parapneumonic effusions. Disease Markers. 2016; 2016 (

24. Hackner K, Errhalt P, Handzhiev S. Ratio of carcinoembryonic antigen in pleural fluid and serum for the diagnosis of malignant pleural effusion. Therapeutic Advances in Medical Oncology. 2019; 11(1758835919850341.

25. Yang Y, Liu Y-L, Shi H-Z. Diagnostic accuracy of combinations of tumor markers for malignant pleural effusion: an updated meta-analysis. Respiration. 2017; 94(1): 62-69.

26. Shen Y, Liang Y, Cheng X, Lu W, Xie X, Wan X. Ovarian fibroma/ fibrothecoma with elevated serum CA125 level: A cohort of 66 cases. Medicine. 2018; 97(34):

27. Antonangelo L, Sales R, Corá A, Acencio M, Teixeira L, Vargas F. Pleural fluid tumour markers in malignant pleural effusion with inconclusive cytologic results. Current Oncology. 2015; 22(5): e336.

28. Dehkordi FS, Valizadeh Y, Birgani TA, Dehkordi KG. Prevalence study of Brucella melitensis and Brucella abortus in cow's milk using dot enzyme linked immuno sorbent assay and duplex polymerase chain reaction. J Pure Appl Microbiol. 2014;8(2):1065-9.

Received: 30 February 2021

Accepted: 15 May 2021 